\title{
Réduire les émissions de particules des véhicules diesel par l'utilisation de biodiesel ${ }^{\text {is }}$
}

\author{
Kristell Guizouarn ${ }^{*}$ a \\ Groupe Avril, 11, rue de Monceau CS 600003, 75378 Paris Cedex 08, France
}

Reçu le 2 septembre 2019 - Accepté le 19 novembre 2019

\begin{abstract}
Résumé - Les véhicules diesel ont un rendement énergétique plus élevé, mais ils produisent davantage de particules que les moteurs à essence ordinaires. Il existe deux moyens efficaces de réduire considérablement les émissions de particules du moteur diesel : le filtre à particules, et l'utilisation de biodiesel. En effet, la composition du carburant a un impact direct sur la combustion, et donc sur les émissions de polluants. Une teneur élevée en biodiesel permet de réduire le nombre de particules produites. Plus la quantité de biodiesel dans le carburant mélangé est importante, moins des particules sont émises. Lorsque les technologies de filtre et de biodiesel sont combinées, les émissions de particules sont quasi-inexistantes.
\end{abstract}

Mots clés : biocarburant / biodiesel / diesel / émissions polluantes / pollution de l'air / particules fines

\begin{abstract}
Reduce particulate emissions from diesel vehicles through the use of biodiesel. Diesel vehicles have higher fuel efficiency however they produce more particles than common gasoline motors. There are two efficient ways to drastically reduce those particulates emission from diesel motor. First particles filter, second biodiesel uses. Indeed the fuel composition has a direct impact on combustion, and therefore on pollutant emissions. A high content of biodiesel makes it possible to reduce the number of particles produced. The more the content of biodiesel in the fuel mixed, the less particles are emitted. When both filter and biodiesel technologies are used together the particles emission are almost inexistent.
\end{abstract}

Keywords: biofuel / biodiesel / diesel / particles / air pollution / particles filter

Il y a deux façons de réduire l'émission de particules pour les moteurs diesel : équiper les véhicules de filtres à particules (FàP) et incorporer du biodiesel dans le carburant.

Depuis 2011 (norme Euro 5 obligatoire, Règlement (CE) $\left.\mathrm{n}^{\mathrm{o}} 715 / 2007,2007\right)$, toutes les voitures particulières neuves à moteur Diesel sont équipées de FàP. Ces filtres, particulièrement efficaces, permettent de produire une teneur en particules en sortie du pot d'échappement quasi similaire à celle de l'air ambiant.

La composition du carburant (en particulier sa teneur en oxygène) a quant à elle un impact direct sur la combustion, donc sur les émissions de polluants. Ainsi, une teneur plus élevée en biodiesel permet naturellement de réduire le nombre et la masse de particules produites.

Afin de disposer de données actuelles selon les normes européennes en vigueur et à venir, la filière oléo-protéagineuse

\footnotetext{
H Contribution to the Topical Issue "Biodiesel / Biodiesel".

*Correspondance : kristell.guizouarn@groupeavril.com

${ }^{a}$ Directrice Énergies Nouvelles et Affaires Européennes chez Groupe Avril.
}

via le fonds d'action stratégique des oléagineux-protéagineux (FASO), a confié une étude à l'IFPEN en juillet 2016 intitulée : «évaluation de l'impact du biodiesel sur les émissions polluantes et particulièrement sur les suies pour des véhicules légers».

\section{Matériel et méthode}

\subsection{Matrice carburant}

L'objectif de cette étude était de dresser un constat sur le niveau des émissions polluantes et particulièrement sur les particules lors de l'utilisation de gazole dans lequel est ajouté du biodiesel à hauteur de 8,10 et $30 \% \mathrm{v} / \mathrm{v}$, en comparaison à du gazole sans biodiesel (Tab. 1).

Le carburant B0 est un gazole EN590 ne contenant pas d'ester; il sera utilisé comme référence pour tous les résultats présentés mais aussi comme carburant de base pour les mélanges avec l'ester.Le carburant $\mathrm{B} 8$ correspond à un ajout de $8 \% \mathrm{v} / \mathrm{v}$ d'ester (EMC - Ester méthylique de colza) dans le B0.

Le carburant B10 correspond à un ajout de $10 \% \mathrm{v} / \mathrm{v}$ d'EMC dans le B0. 
Tableau 1. Matrice carburant complète avec formulation de chaque carburant.

\begin{tabular}{ll}
\hline Nom du carburant & Formulation \\
\hline B0 & Carburant de référence sans ester \\
B8 & B0 $+8 \% \mathrm{v} / \mathrm{v}$ EMC \\
B10 & B0 $+10 \%$ v $/ \mathrm{v}$ EMC \\
B30 & B0 $+30 \% \mathrm{v} / \mathrm{v}$ EMC \\
\hline
\end{tabular}

Le carburant $\mathrm{B} 30$ correspond à un ajout de $30 \% \mathrm{v} / \mathrm{v}$ d'EMC dans le B0.

\subsection{Choix de véhicules}

Deux véhicules ont été testés, l'un représentant une technologie récente de type Euro 6 avec FàP (Peugeot 3081.6 BlueHDi 120 ch FAP BVM6) et l'autre une technologie plus ancienne avec un véhicule non muni de FàP (Renault Laguna 2007 DCI Privilège).

Ce choix repose sur une recherche approfondie préalable visant à connaître l'état actuel du parc automobile en circulation en France. Le choix de ces véhicules a été fait pour être représentatif des véhicules composant le parc roulant français actuel en se basant sur des études réalisées par l'IFSTTAR - Institut français des sciences et technologies des transports, de l'aménagement et des réseaux - (André et al., 2014), par le CEREMA - Centre d'études et d'expertise sur les risques, l'environnement, la mobilité et l'aménagement(Matheus et al., 2016) et le CCFA (2016)-Comité des constructeurs français d'automobiles - et qui donnent une vision macroscopique du parc français. $\grave{A}$ cette date, les motorisations Euro $5(31 \%)$ et Euro $4(25 \%)$ étaient les plus répandues en circulation, l'âge moyen des véhicules en circulation étant de 7 à 8 ans. De fait en France, plus de $50 \%$ du parc de véhicules légers roulant ne sont pas équipés de FàP.

\subsection{Moyens d'essais}

Les essais véhicules ont été réalisés sur les bancs à rouleaux d'IFPEN Rueil. Les caractéristiques principales du banc 07 sur lequel ont été réalisés les essais sont présentées dans la figure 1 .

Un banc à rouleaux permet de reproduire le fonctionnement d'un véhicule sur un profil routier préalablement défini. Il est constitué ici d'un châssis supportant deux rouleaux eux-mêmes entraînés ou freinés, d'une part par les roues motrices du véhicule, d'autre part par un système d'absorption d'énergie reproduisant la résistance à l'avancement. Un dispositif approprié simule l'effet de la masse du véhicule en reproduisant son inertie pendant les variations de vitesse. Une ventilation asservie à la vitesse du véhicule permet de reproduire le refroidissement naturel du radiateur moteur situé en face avant du véhicule. Le contrôle et l'acquisition des paramètres du banc sont assurés par le logiciel Morphée développé par D2t.

Les bancs à rouleaux d'IFPEN Rueil sont équipés d'un tunnel de dilution à volume constant (CVS) permettant la réalisation d'essais véhicules dans des conditions normalisées et les mesures de polluants gazeux et particulaires associées,

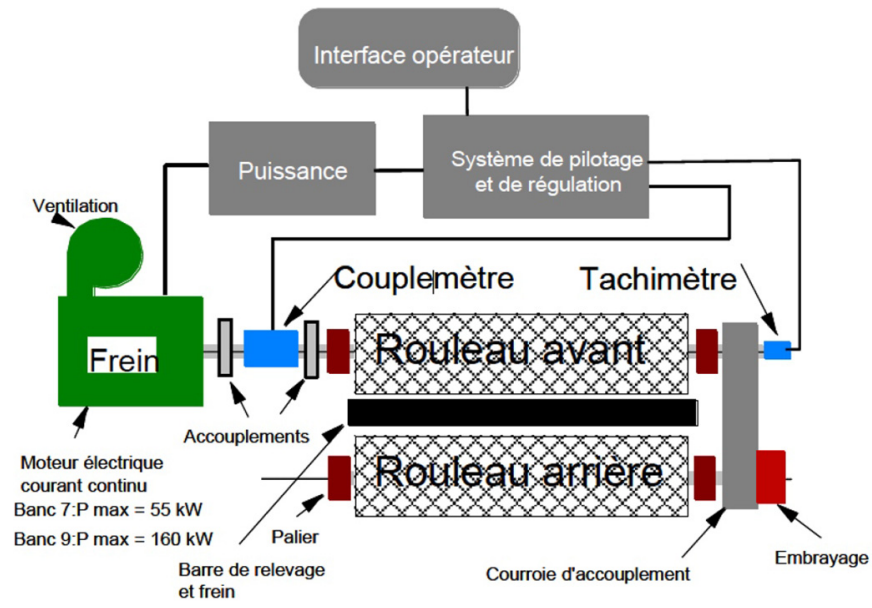

Fig. 1. Schéma de principe du banc à rouleaux IFPEN Rueil.

satisfaisant les normes européennes. Ils sont équipés d'une baie d'analyse des gaz dilués, ainsi que de systèmes de mesure de la masse et du nombre de particules, satisfaisant notamment le protocole PMP. Le principe du CVS repose sur un prélèvement à volume constant et taux de dilution variable. L'air de dilution utilisé au niveau de celui-ci est régulé en température et hygrométrie sur le banc. La réalisation d'essais normalisés impose des périodes de conditionnement du véhicule avant essais qui réduisent d'autant la durée et le nombre d'essais réalisables.

Les informations relatives au banc à rouleaux et aux conditions ambiantes sont acquises à une fréquence de $1 \mathrm{~Hz}$ : température ambiante, pression atmosphérique et humidité relative dans le banc. Les informations relatives aux véhicules: vitesse théorique et réelle du véhicule, débit d'air du moteur, richesse échappement, ainsi que les polluants gazeux réglementés mesurés par les baies 5 gaz du banc à rouleau $\left(\mathrm{O}_{2}, \mathrm{CO}, \mathrm{CO}_{2}, \mathrm{NO}_{x}, \mathrm{HC}\right)$, et la mesure de $\mathrm{CO}_{2}$ en aval du FPS4000 , dédiée au calcul du taux de dilution réel, sont eux aussi acquis à la fréquence de $1 \mathrm{~Hz}$.

L'émission de particules des véhicules est mesurée avec un DMS-500 (Cambustion) qui donne accès à leur répartition granulométrique et par classe de taille sur l'ensemble d'un cycle de roulage.

\subsection{Caractérisation des carburants}

La caractérisation des carburants a été réalisée sur tous les carburants afin de pouvoir relier les émissions polluantes constatées aux propriétés physico-chimiques et à la composition de chaque échantillon.

Les analyses suivantes seront réalisées sur les carburants diesel : densité, analyse élémentaire $(\mathrm{C}, \mathrm{H}, \mathrm{O})$, pouvoir énergétique, HAP - Hydrocarbures aromatiques polycycliques-, distillation et composition chimique détaillée par chromatographie 2D.

La GCxGC est une technique de plus en plus utilisée pour les échantillons possédant une composition chimique complexe. Deux colonnes de séparation sont utilisées, la $1^{\text {re }}$ colonne est apolaire, et les composés sont séparés par leur volatilité. Ensuite, chaque élution, c'est-à-dire la fraction déjà séparée par la $1^{\mathrm{re}}$ colonne passe dans la $2^{\mathrm{e}}$ colonne, qui est polaire, et sépare 


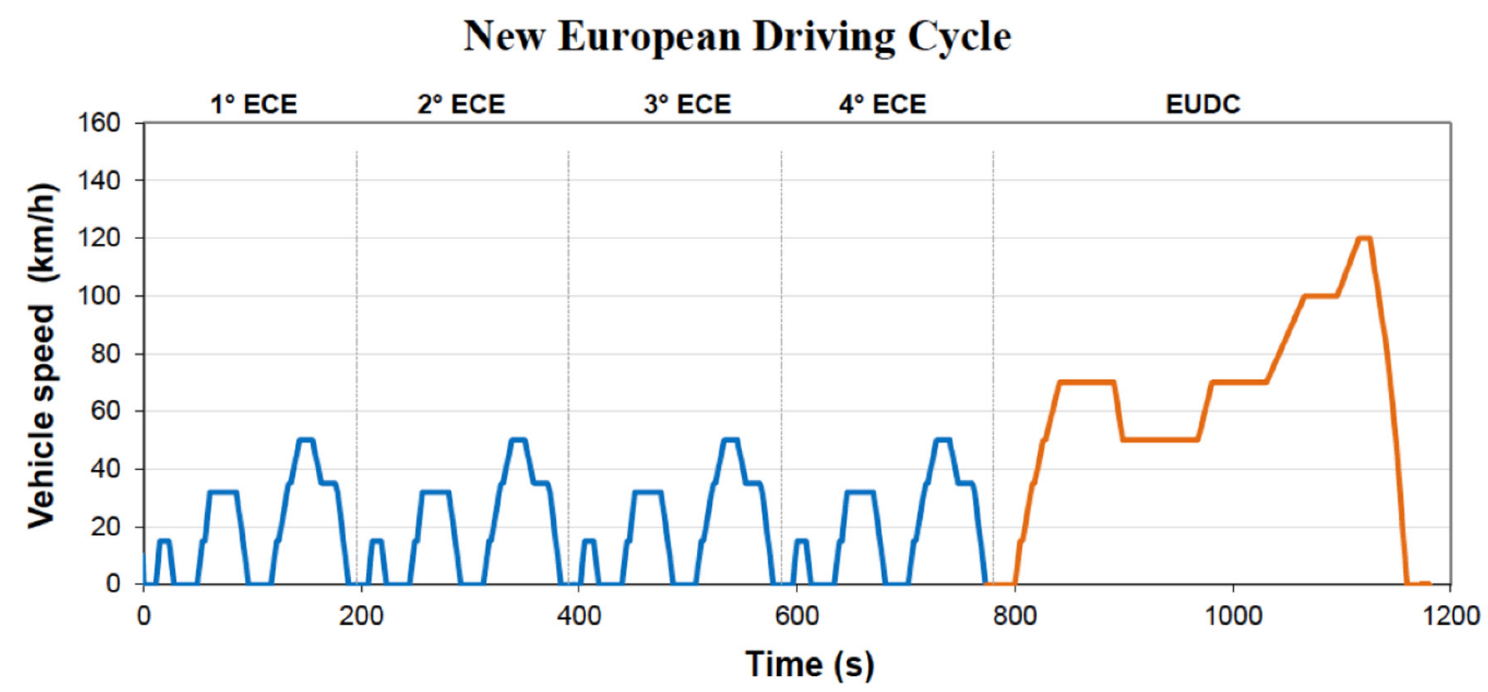

Fig. 2. Cycle NEDC.

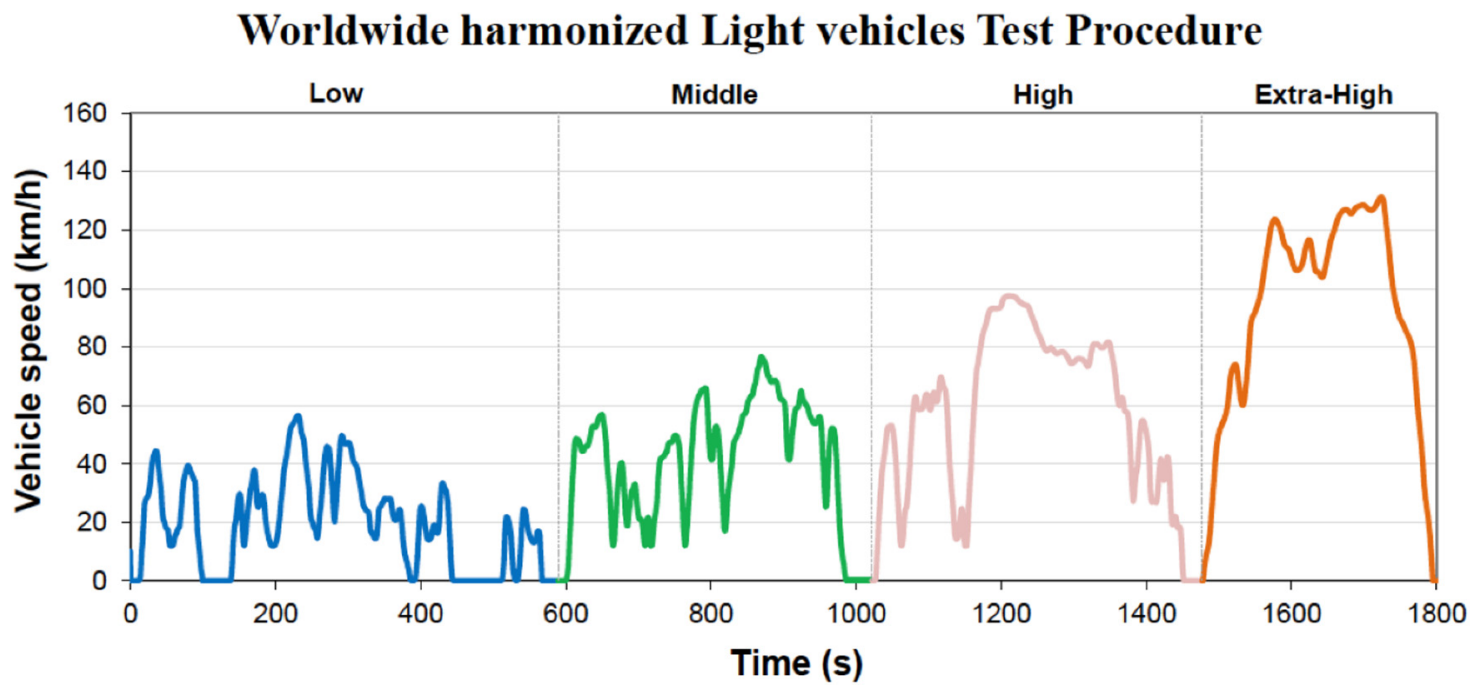

Fig. 3. Cycle WLTC.

donc dans ce cas les composés par leur polarité (par exemple la séparation se fait entre les composés saturés avec chaînes linéaires ainsi que branchées, les cycliques, les aromatiques, les di-aromatiques, les hétéroatomes, etc.).

\subsection{Deux cycles d'essais}

Deux cycles différents ont été réalisés :

- Cycle NEDC (New European Driving Cycle, Fig. 2): Cycle européen actuel d'homologation utilisé au sein de la communauté Européenne pour les véhicules légers. Il permet de dresser un constat vis à vis des seuils d'émissions réglementaires actuels. Ce cycle est constitué de quatre phases «urbaines» (Fig. 4; du $1^{\mathrm{er}} \mathrm{ECE}$ au $4^{\mathrm{e}}$ ECE) à vitesse réduite, suivies d'une phase «extra urbaine» (EUDC) au cours de laquelle une vitesse maximale de $120 \mathrm{~km} / \mathrm{h}$ est atteinte. La distance totale parcourue durant l'ensemble du cycle est de $11007 \mathrm{~km}$, pour une durée totale de $1180 \mathrm{~s}$. La succession de plusieurs paliers à vitesse stabilisée et des transitoires de charge assez progressifs permet de garantir une bonne reproductibilité des mesures d'émissions polluantes. Néanmoins, ce cycle est considéré comme peu représentatif des conditions de roulage réelles :

- Cycle WLTC (Worldwide harmonized Light vehicles Test Cycle, Fig. 3): futur cycle qui remplace le cycle NEDC à compter de la date d'application de la future norme Euro $6 c$, à partir de 2017. Son objectif est d'harmoniser les procédures d'homologation à travers le monde, mais également d'être plus représentatif des conditions d'utilisation réelles. Le WLTC se compose de quatre phases nommées «low», « middle», « high » et « extra-high ». La maximale atteinte au cours du cycle est $130 \mathrm{~km} / \mathrm{h}$, la distance totale parcourue durant cycle est $23262 \mathrm{~km}$, pour 


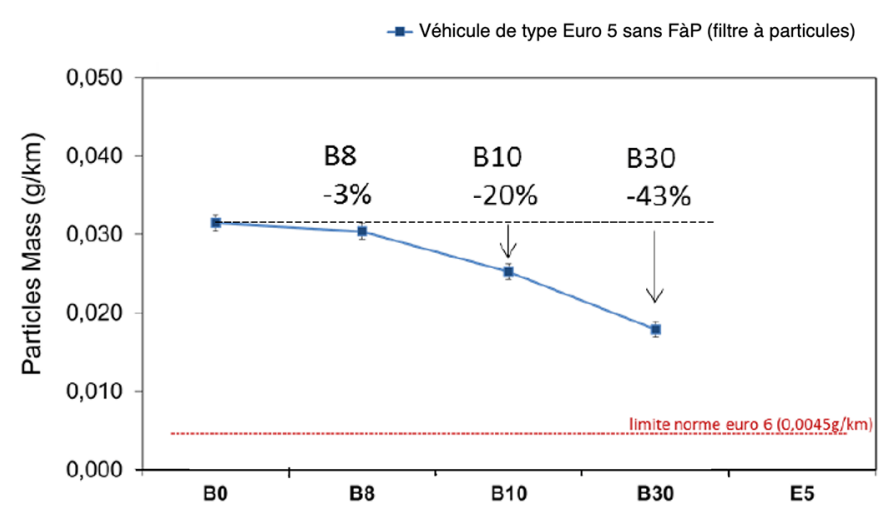

Fig. 4. Émission de particules en masse d'un véhicule Euro 5 sans FàP en fonction de la matrice carburant (B0, B8, B10, B30), sur le cycle NEDC.

une durée totale de $1800 \mathrm{~s}$. Ce cycle se différencie du cycle NEDC disparition des phases stabilisées et par l'augmentation de la part des phases transitoires.

\subsection{Protocole d'essai et répétabilité}

Le protocole pour les essais réalisé est le suivant:

- conditionnement du véhicule selon la norme;

- macération du véhicule entre 20 et $25^{\circ} \mathrm{C}$;

- essai sur cycle NEDC ou WLTC.

À chaque essai, sont mesurées les émissions de $\mathrm{CO}, \mathrm{HC}$, $\mathrm{NO}_{x}$, particules (masse) sur le cycle par prélèvement en sac et sur filtres Pallflex ainsi que les émissions de $\mathrm{CO}, \mathrm{HC}, \mathrm{NO}_{x}$, $\mathrm{CO}_{2}$ et particules (nombre) mesurés en continu dans les gaz dilués. Les émissions massiques normalisées à partir de l'analyse des sacs sont exprimées en $\mathrm{g} / \mathrm{km}$ et les émissions massiques en gaz dilué (instantanées), exprimées en $\mathrm{g} / \mathrm{s}$.

La répétabilité est évaluée dans un premier temps sur deux essais et sur la base du $\mathrm{CO}_{2}$ en appliquant la formule suivante :

$$
\text { Répétabilité }=\frac{2 \times \sigma_{\mathrm{CO}_{2}}}{\text { Moyenne } \times \sqrt{\text { Nbessais }}}, \text { avec } \sigma_{\mathrm{CO}_{2}} \text { l'écart-type }
$$
des mesures de $\mathrm{CO}_{2}$ global.

Une valeur supérieure à $1 \%$ traduit une mauvaise répétabilité, et dans ce cas, un troisième essai est réalisé. L'essai s'écartant des deux autres sera alors non exploité. À noter que cette démarche est similaire à celle de l'UTAC sauf que l'UTAC calcule une répétabilité sur 3 essais et pour chaque polluant.

Les résultats présentés dans ce rapport correspondent donc à la moyenne des deux essais rentrant dans les critères de répétabilité.

\section{Résultats}

\subsection{Analyse élémentaire, densité, HAP, PCI}

Les résultats de l'analyse élémentaire $(\mathrm{C}, \mathrm{H}, \mathrm{O})$, densité, HAP et pouvoir calorifique inférieur et supérieur pour le gazole et biodiesel sont présentés dans le tableau 2 .

Comme attendu, la densité augmente très légèrement avec l'ajout du biodiesel, le B30 présente une densité de 0,8505
Tableau 2. Résultats de densité, analyse élémentaire, HAP et pouvoir calorifique des carburants.

\begin{tabular}{lcccc}
\hline & B0 & B8 & B10 & B30 \\
\hline Densité $\left(\mathrm{g} / \mathrm{cm}^{3}\right)$ & 0,836 & 0,841 & 0,841 & 0,851 \\
$\mathrm{C}(\% \mathrm{~m} / \mathrm{m})$ & 86,2 & 85,4 & 85,2 & 82,6 \\
$\mathrm{H}(\% \mathrm{~m} / \mathrm{m})$ & 13,49 & 13,45 & 13,43 & 12,99 \\
O $(\% \mathrm{~m} / \mathrm{m})$ & 0 & 0,97 & 1,24 & 3,54 \\
$\mathrm{~N}(\% \mathrm{~m} / \mathrm{m})$ & 0,07 & 0,06 & 0,16 & 0,05 \\
HAP $(\% \mathrm{~m} / \mathrm{m})$ & 6,4 & 5,7 & 5,6 & 5,4 \\
PCI $(\mathrm{MJ} / \mathrm{kg})$ & 43,3 & 42,5 & 42,3 & 41,1 \\
PCI $(\mathrm{MJ} / \mathrm{L})$ & 36,2 & 35,7 & 35,6 & 34,9 \\
PCS $(\mathrm{MJ} / \mathrm{kg})$ & 45,8 & 45,1 & 45,3 & 43,9 \\
Ethanol & & & & \\
ETBE & & & & \\
MTBE & 1,88 & 1,89 & 1,89 & 1,88 \\
Ratio H/C & & & & \\
\hline
\end{tabular}

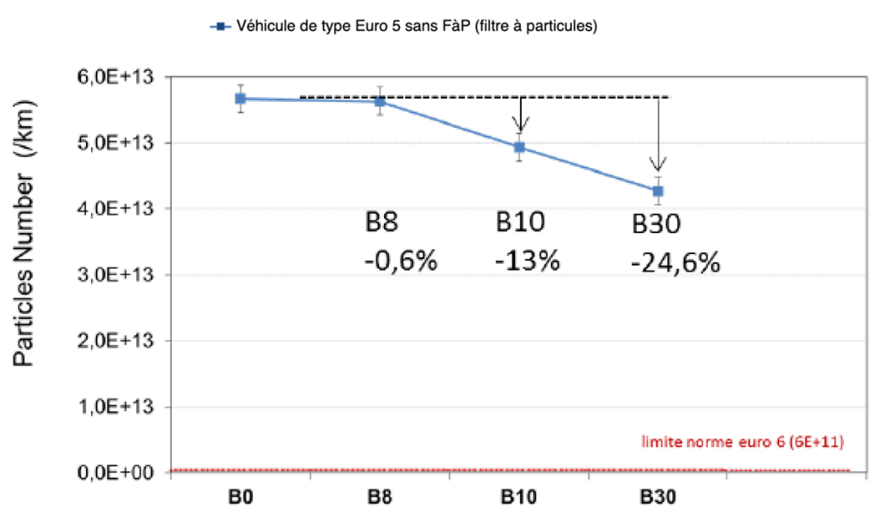

Fig. 5. Émission de particules en nombre d'un véhicule Euro 5 sans FàP en fonction de la matrice carburant $(\mathrm{B} 0, \mathrm{~B} 8, \mathrm{~B} 10, \mathrm{~B} 30)$, sur le cycle NEDC.

alors que pour le B0 elle est de $0,8358 \mathrm{~g} / \mathrm{cm}^{3}$ soit $\sim 2 \%$ supérieur seulement mais significatif tout de même. Le B8 et le $\mathrm{B} 10$ ont une densité très proche. Par rapport à l'analyse élémentaire, les résultats sont cohérents, plus la quantité d'EMC est importante plus la quantité d'oxygène et hydrogène augmente, et le carbone diminue.

Le PCI (Pouvoir calorifique inférieur) du biodiesel B30 est environ $5 \%$ inférieur à celui du $\mathrm{B} 0$. Le $\mathrm{B} 8$ et le $\mathrm{B} 10$ présentent un PCI seulement $\sim 1,5 \%$ inférieur au B0. Les différences observées sont assez faibles et dans la marge d'erreur des mesures.

\section{2 Émissions de particules}

Les émissions en masse soulignent que l'impact de l'ester est notable avec une diminution des particules très importante avec l'augmentation de la teneur en biocarburant sur le véhicule de type Euro 5 sans FàP: sur le cycle NEDC en vigueur, la réduction atteint $-43 \%$ entre le $\mathrm{B} 0$ et le $\mathrm{B} 30$ (Fig. 4) et pour le WLTC, $-24 \%$. Le profil d'évolution du nombre de particules en fonction du carburant met pour sa part en évidence une réduction d'environ $25 \%$ pour le cycle NEDC 
(Fig. 5) et de $-14 \%$ pour le WLTC sur ce même véhicule, entre le B0 et le B30.

Les résultats sur les autres polluants $\left(\mathrm{CO}, \mathrm{HC}, \mathrm{NO}_{x}, \mathrm{CO}_{2}\right)$ pour les deux véhicules et sur les deux cycles sont détaillés dans la section 3 .

\section{Discussion}

Le travail mené dresse donc un constat global sur l'impact que l'ajout de biodiesel joue sur les émissions polluantes $\left(\mathrm{CO}, \mathrm{HC}, \mathrm{NO}_{x}, \mathrm{CO}_{2}\right.$, et particules) lors d'essais réalisés sur un banc à rouleaux. La matrice carburant est constituée des carburants suivants : B8, B10 et B30, avec le carburant B0, comme carburant de référence. Deux véhicules diesel (l'un Euro 6 équipé de FàP, l'autre Euro 5 sans FàP) ont été testés.

\section{1 Émissions de CO}

La limite d'émissions de CO pour la norme Euro 6 diesel est de $0,5 \mathrm{~g} / \mathrm{km}$; la marge d'erreur dans la mesure pour le cycle NEDC qui est de $\pm 0,02 \mathrm{~g} / \mathrm{km}$, obtenu lors des essais circulaires entre plusieurs laboratoires. Dans ces essais, il n'est pas possible de dégager une tendance claire entre les biocarburants. En plus avec la marge d'erreur à $0,02 \mathrm{~g} / \mathrm{km}$ les écarts observés ne sont pas significatifs. Dans tous les cas, pour tous les voitures et tous les carburants, les émissions de CO sur le cycle NEDC sont dans les limites imposées par la norme Euro $6 \mathrm{~b}$ diesel.

\section{2 Émissions de HC}

La limite d'émissions de HC pour la norme Euro 6 des véhicules essence est de $0,1 \mathrm{~g} / \mathrm{km}$, pour les véhicules diesel la norme Euro 6 englobe $\mathrm{HC}$ et $\mathrm{NO}_{x}$ avec une limite à $0,170 \mathrm{~g} /$ $\mathrm{km}$. La marge d'erreur dans la mesure pour l'HC en cycle NEDC de $\pm 0,002 \mathrm{~g} / \mathrm{km}$, obtenu lors des essais circulaires entre plusieurs laboratoire. Pour le cycle NEDC aucune tendance peut être observée. Les valeurs sont soit identiques soit dans la marge d'erreur de la mesure pour tous les carburants. Toutefois pour le cycle WLTC, on note une diminution mineure des émissions d'HC avec l'augmentation de la teneur en biocarburant. Les valeurs diminuent de $0,01 \mathrm{~g} / \mathrm{km}$ pour le $\mathrm{B} 0$ et le $\mathrm{B} 8$ à $-50 \%$ pour le $\mathrm{B} 10(0,005 \mathrm{~g} / \mathrm{km})$ et arrive à $0,001 \mathrm{~g} / \mathrm{km}$ pour le $\mathrm{B} 30$. Comme pour le $\mathrm{CO}$, dans les deux cas, tous les véhicules diesel respectent largement le niveau d'émission de la norme en vigueur et future sur les émissions : plus de 10 fois inférieur à la norme Euro 6 .

\section{3 Émissions de $\mathrm{NO}_{x}$}

Les émissions de $\mathrm{NO}_{x}$ sont limitées à $0,18 \mathrm{~g} / \mathrm{km}$ en Euro 5 et $-0,08 \mathrm{~g} / \mathrm{km}$ en Euro 6 pour les véhicules diesel. La marge d'erreur de la mesure pour les $\mathrm{NO}_{x}$ en cycle NEDC de $\pm 0,006 \mathrm{~g} / \mathrm{km}$, obtenue lors des essais circulaires entre plusieurs laboratoires européens. Les deux véhicules diesel dépassent les émissions pour lesquelles ils ont été homologués et ce pour les deux cycles, NEDC et WLTC. Par rapport à l'effet du biodiesel, les émissions semblent augmenter très
Tableau 3. Réductions des différents polluants obtenues grâce à l'utilisation de carburant à base de biodiesel, selon leur teneur, sur un véhicule représentatif du parc roulant (Euro 5 sans FàP).

\begin{tabular}{llcr}
\hline B0 (Référence) & B8 & B10 & B30 \\
\hline $\mathrm{CO}$ & $=(\mathrm{NS})$ & $=(\mathrm{NS})$ & $=(\mathrm{NS})$ \\
$\mathrm{HC}$ & $=(\mathrm{NS})$ & $=(\mathrm{NS})$ & $=(\mathrm{NS})$ \\
$\mathrm{NO}_{x}$ & $=(\mathrm{NS})$ & $=(\mathrm{NS})$ & $=(\mathrm{NS})$ \\
$\mathrm{CO}_{2}$ & $==(\mathrm{NS})$ & $=(\mathrm{NS})$ & $=(\mathrm{NS})$ \\
Émissions de particules en nombre & & & \\
Cycle NEDC & $-0,6 \%$ & $-13 \%$ & $-24,6 \%$ \\
Cycle WLTC & $==(\mathrm{NS})$ & $-4,5 \%$ & $-14 \%$ \\
Émissions de particules en masse & & & \\
Cycle NEDC & $-3 \%$ & $-20 \%$ & $-43 \%$ \\
Cycle WLTC & $-2,5 \%$ & $-11 \%$ & $-24 \%$ \\
\hline
\end{tabular}

légèrement avec l'ajout de l'ester. Néanmoins, l'augmentation est significative seulement dans le cas du B30 pour les deux véhicules diesel sur le cycle WLTC.

\section{4 Émissions de $\mathrm{CO}_{2}$ et consommation}

Les émissions de $\mathrm{CO}_{2}$ ne sont pas réglementées. La marge d'erreur est de $\pm 0,8 \mathrm{~g} / \mathrm{km}$. Une légère diminution des émissions est observée entre le B0 et le B8 pour les deux véhicules. Pourtant entre le B8, B10 et B30 les valeurs remontent et ceux du $\mathrm{B} 30$ sont équivalents à ceux du $\mathrm{B} 0$ initial. Pour le cycle WLTC, les mêmes constats peuvent être faits : diminution des émissions entre B0 et B8 suivi d'une augmentation avec l'ajout du EMC. Néanmoins, les émissions avec le $\mathrm{B} 30$ restent inférieures à celles du $\mathrm{B} 0$. On peut donc difficilement conclure à un effet du biodiesel sur les émissions de $\mathrm{CO}_{2}$. Enfin, il n'y a pas de surconsommation de carburant engendrée par l'ajout de biodiesel EMC.

\section{5 Émissions de particules}

La norme Euro 6 limite les émissions de particules en masse à $0,0045 \mathrm{~g} / \mathrm{k}$; les émissions de particules en nombre sont réglementées depuis la norme Euro 5b. Les essais montrent que la diminution des particules peut aller jusqu'à $43 \%$ en masse et jusqu'à $24,6 \%$ en nombre pour un carburant B30 sur un véhicule non équipé de FàP.

Ainsi, les résultats obtenus sur banc à rouleaux ne montrent aucune augmentation ou diminution des émissions de $\mathrm{CO}, \mathrm{HC}$ et $\mathrm{NO}_{x}$ lorsque l'on ajoute du biodiesel au carburant. En revanche, les émissions de particules, qui dépassent très largement les normes avec du $\mathrm{B} 0$, diminuent très nettement en masse et en nombre lorsque la teneur en biodiesel augmente pour le véhicule diesel non muni de FàP. Le biodiesel réduit les émissions en amont du filtre et donc a un impact positif sur le fonctionnement du filtre. Le tableau 3 résume les réductions des différents polluants obtenues grâce à l'utilisation de carburant à base de biodiesel, selon leur teneur.

La présence d'oxygène dans le biodiesel permet d'améliorer la qualité de la combustion et d'entraîner une diminution des polluants. Cela est clairement démontré pour les particules 
dont le nombre peut être réduit de manière significative, ainsi que pour les fumées noires. Plus le taux d'incorporation de biodiesel est élevé, plus l'impact est important, autrement dit plus la diminution des particules est forte. Ainsi, l'incorporation de biodiesel à $30 \%$ dans les gazoles conduit à une réduction significative des émissions de pollution locale. L'impact d'une teneur croissante en biodiesel est particulièrement efficace sur les véhicules non équipés de FàP car le biocarburant agit en amont de l'échappement.

\section{Conclusion}

Une motorisation diesel performante, un FàP et la présence de biodiesel dans le gazole sont autant d'éléments qui constituent une réponse efficace et adaptée aux enjeux de pollution locale liée au transport routier.

La tendance forte vers la réduction de la consommation de carburant des véhicules par le biais de technologies innovantes ainsi qu'une politique nationale assurant une incorporation garantie de biodiesel dans le gazole à hauteur de près de $10 \%$ permettent également de réduire les émissions de $\mathrm{CO}_{2} \mathrm{du}$ transport. Cela a un effet direct sur la pollution globale et donc dans la lutte contre le réchauffement climatique.

\section{Références}

André M, Roche AL, Bourcier L. 2014. Statistiques de parcs et trafic pour le calcul des émissions de polluants des transports routiers pour la France (janvier 2013, révision mars 2014). Rapport IFSTTAR-LTE, Bron, France, 133 p. Disponible sur https://hal.archives-ouvertes.fr/hal-01431629 (dernière consult. : 28/10/2019).

Comité des constructeurs français d'automobiles. 2016. Le marché automobile français (plus disponible en ligne).

Matheus J, Guével V, Le Roy E (Cerema). 2016. Étude sur les parcs roulants routiers français. Inventaire, comparaison et impact sur les courbes d'émission. Cerema, direction techniques insfrastructures et matériaux, $77 \mathrm{p}$. Disponible sur http://www.bv. transports.gouv.qc.ca/mono/1179446.pdf (dernière consult. : 28/ 10/2019).

Règlement (CE) $n^{\circ} 15 / 2007$. 2007. Règlement (CE) $n^{\circ} 715 / 2007 d u$ Parlement européen et du Conseil du 20 juin 2007 relatifà la réception des véhicules à moteur au regard des émissions des véhicules particuliers et utilitaires légers (Euro 5 et Euro 6) et aux informations sur la réparation et l'entretien des véhicules (Texte présentant de l'intérêt pour l'EEE). Disponible sur https://eur-lex.europa.eu/legalcontent/FR/TXT/?uri=celex:32007R0715 (dernière consult. : 14/10/ 2019).

Citation de l'article : Guizouarn K. 2020. Réduire les émissions de particules des véhicules diesel par l'utilisation de biodiesel. OCL 27: 11. 